\title{
Knowledge, Attitude and Practices on Diabetes Among Type 2 Diabetic Patients in Iran: A Cross-Sectional Study
}

\author{
Shooka Mohammadi ${ }^{1,2}$, Norimah A. Karim ${ }^{1, ~ *, ~ R u z i t a ~ A b d ~ T a l i b ~}{ }^{1}, \operatorname{Reza}_{\text {Amani }}{ }^{2}$ \\ ${ }^{1}$ Nutritional Sciences Programme, School of Healthcare Sciences, Faculty of Health Sciences, Universiti Kebangsaan Malaysia, Kuala Lumpur, \\ Malaysia \\ ${ }^{2}$ Department of Nutrition and Dietetics, Faculty of Para Medicine, Ahvaz Jundishapur University of Medical Sciences, Ahvaz, Iran
}

Email address:

Shooka.mohammadi@gmail.com (S. Mohammadi),norimahkarim@ukm.edu.my (N. A. Karim), rzt@ukm.edu.my (R. A. Talib), rezaamani@hotmail.com (R. Amani)

\section{To cite this article:}

Shooka Mohammadi, Norimah A. Karim, Ruzita Abd Talib, Reza Amani. Knowledge, Attitude and Practices on Diabetes Among Type 2 Diabetic Patients in Iran: A Cross-Sectional Study. Science Journal of Public Health. Vol. 3, No. 4, 2015, pp. 520-524.

doi: $10.11648 /$ j.sjph.20150304.20

\begin{abstract}
Following fast increase in the incidence of type 2 diabetes among Iranians, diabetes self-care activities are important. This cross-sectional study was carried out to evaluate the knowledge, attitude and practices regarding diabetes among 100 Iranian type 2 diabetics (61 female, 39 male) aged between 37 to 66 years, attending the out-patient Diabetes Clinic at Golestan Hospital, Ahvaz, Iran. A face-to-face interview was done and a validated knowledge, attitude and practice (KAP) questionnaire was used to determine knowledge, attitude and practices of diabetes patients. The mean duration of diabetes among patients was $4.05 \pm 1.4$ years. Fifty three percent of patients had good glycemic control $(\mathrm{HbA} \mathrm{c}$ level $(=<7 \%))$. The results showed that attitude related to diabetes was acceptable in the majority; while knowledge and self-care practices were insufficient. The overall mean $( \pm$ SD) score of the knowledge, attitude and practice of patients based on KAP questionnaire was in medium level $(17.37 \pm 2.23)$ in which total maximum possible score was 29 . KAP score was low for $9 \%$ of patients. There was significant association between low level of education (primary) or not having formal education with poor KAP score about diabetes compare to higher level of education [illiterate: $\mathrm{OR}=2.6,95 \% \mathrm{CI}=(0.23-30.80)$, primary: $\mathrm{OR}=0.23,95 \%$ $\mathrm{CI}=(0.43-1.27), \mathrm{P}<0.05]$. While there was no significant association between the KAP scores and HbA1c level. As the consequence of low awareness of diabetes among patients affects their ability of self-care management and lead to negative impact on outcome of diabetes. Patients need to be educated on how to prevent diabetes complications. Health care providers should promote self-care activities in the process of diabetes education.
\end{abstract}

Keywords: Knowledge, Attitude, Practices, Type 2 Diabetes Patients, Iran

\section{Introduction}

Diabetes is a common chronic illness in almost all countries [1]. Throughout the last twenty years, the incidence of diabetes has been raised intensively in many parts of the world [2]. It is estimated that there are nearly 346 diabetes patients globally [3]. In Asia, the rise in prevalence of type 2 diabetes is even more distressing with increased occurrence among young adults [4]. A national survey in Iran indicated that the incidence to be $7.7 \%$ in adults $25-65$ years [5]. Its prevalence is predicted to increase to 5.1 million by 2025 [6]. Lifestyle and environmental factors are the main causes of the extreme increase in the incidence of type 2 diabetes [7]. This rapidly-rising prevalence among developing countries is recognized to be the effects of urbanization $[8,9]$.

Diabetes may increase the risk of microvascular and macro vascular complications and early death in the general population and lead to a vast economic problem for society $[10,11]$. However, by the existence of high prevalence of diabetes, diabetes awareness and management still are major challenges in Iran. Adverse attitudes and psychological problems such as depression are common among diabetes patients and can led to poor diabetes management $[12,13]$.

Very few studies $[14,15]$ have been conducted in Iran to evaluate the level of awareness, attitudes and practices among type 2 diabetic patients. Therefore, this study 
evaluated the knowledge, attitude and practices on diabetes among Iranian type 2 diabetic patients to assess their needs for further educational interventions.

\section{Materials and Methods}

This cross-sectional study was carried out to define level of knowledge, attitude and practices regarding diabetes among 100 type 2 diabetes patients attending to the diabetes clinic in Golestan hospital in Ahvaz a city in southwest Iran during the study period of August to October 2013. After receiving approval from the Ethics Committee of National University of Malaysia (UKM), Face-to-face interview was done. Subjects were selected from type 2 diabetes patients aged more than 30 years and diagnosed with diabetes more than one year. Upon screening, patients were given an information sheet which explained the purpose of the study, participation was voluntary and they were able to refuse participation in or withdraw from the study. Only the patients who met the inclusion criteria and signed consent form were recruited to participate in this study.

A KAP (Knowledge, Attitude \& practice) questionnaire which was developed and validated by Mukhopadhyay and her colleagues [16] used and pre tested on 10 patients. The reliability of the questionnaire was considered satisfactory after discussion with experts. This modified questionnaire was then used to interview with the type 2 diabetes patients.

Questionnaire consisted of 13 questions on general knowledge of diabetes, eight questions for attitude and eight questions for the practices regarding diabetes self-care and management. Each correct answer was given a score of 'one' and each wrong answer inclusive of ("don't know") was given a score of 'zero'. The maximum possible score for knowledge, attitude and practice of patients were 13, 8 and 8 respectively. Those who scored $75 \%$ and above were considered as having adequate knowledge/good practice and those with a score of $50 \%$ or less is taken as having inadequate knowledge / poor practice, those who racked up between $51-74 \%$ was considered as having moderately adequate knowledge/ practices).

The Statistical Package for Social Sciences (SPSS ${ }^{\circledR}$ ) version 21 and Microsoft excel spreadsheet were used to analyze data in this study. Descriptive statistics including frequency, means and standard deviation were applied to present subject's demographic information and KAP score. Binary logistic regression was used to determine the association between KAP score and level of HbA1c. A multiple logistic regression analysis adjusted with education, age, duration of diabetes, gender $\&$ income was carried out to define the effects of multiple factors on Poor KAP score. The associated $95 \% \mathrm{CI}$, and $\mathrm{P}<0.05$ was considered as the significant level.

\section{Results}

The mean age of men and women was $56 \pm 6.1$ and $53.4 \pm$ 6.7 years respectively. Sixty one percent of diabetes patients were female and $39 \%$ were male with mean duration of diabetes $4.05 \pm 1.4$ years. Almost 27 patients were illiterate, but the majority $(41 \%)$ of them did not educated after primary level. Of the males, 27 patients $(27 \%)$ were employed, $12 \%$ were retired and most of the females $(61 \%)$ were housewives. Majority of patients (72\%) had household incomes lower than 8,000,000 Rials a month (USD282). More than a half of the patients (68\%) reported family history of diabetes. Fifty three percent of patients had good glycemic control $(\mathrm{HbA} 1 \mathrm{c}$ level $=<7 \%)$.

Thirty patients supposed that diabetes characterized by high sugar intake, 16 patients $(14 \%)$ stated that it is due to failure in insulin secretion and $56 \%$ did not have any idea on this matter. All of the patients knew that both genders could be involved with diabetes. Frequent urination was indicated as the most common symptom of diabetes by 81 patients (81\%). Regarding complications of diabetes, awareness of heart disease was stated to be the highest in 72 patients $(72 \%)$ followed by eye disease (17\%), kidney diseases $(6 \%)$ and foot problems in $5 \%$. Healthy diet was indicated to be the most important lifestyle factor that could help in prevention of diabetes. Knowledge of other lifestyle factors was not good (Table 1).

Awareness of hypoglycemia, the need for periodic eye examination and foot care in diabetics were found to be poor which has been shown in Table 2. Distribution of diabetic patients based on the level of knowledge of diabetes is described in Table 3, which is revealed that the majority of diabetic patients had a medium level of knowledge on diabetes. The mean score of knowledge, attitude and practices of diabetic patients has been presented in Table 4 .

There was significant association between low level of education (primary) or not having formal education with poor KAP score about diabetes compared to higher level of education [illiterate: $\mathrm{OR}=2.6,95 \% \mathrm{CI}=(0.23-30.80)$, primary : $\mathrm{OR}=0.23,95 \% \mathrm{CI}=(0.43-1.27), \mathrm{p}<0.05]$. The rest of demographic variables were not associated with level of KAP score based on multiple logistic regression.

Table 1. Knowledge of type 2 diabetes patients about diabetes $(n=100)$.

\begin{tabular}{ll}
\hline Knowledge of diabetes & Responses \% \\
\hline Symptom(s) of diabetes & \\
Frequent urination & $81 \%$ \\
Frequent hunger & $12 \%$ \\
Frequent thirst & $7 \%$ \\
Complication(s) of diabetes & \\
Heart disease & $72 \%$ \\
Kidney disease & $6 \%$ \\
Eye disease & $17 \%$ \\
Foot problems & $5 \%$ \\
Lifestyle factor(s) which can prevent diabetes & \\
Healthy diet & $71 \%$ \\
Regular exercise & $13 \%$ \\
Weight control & $2 \%$ \\
Don't know & $5 \%$ \\
\hline
\end{tabular}

Patients who passed primary level of education or didn't have any formal education 0.23 and 2.6 times respectively more likely to report a low score of diabetes knowledge 
compared to those who passed secondary level of education. There was no significant association between the KAP scores and $\mathrm{HbA} 1 \mathrm{C}$ level based on binary logistic regression (OR $=1.10,95 \% \mathrm{CI}=(0.92-1.32), P=0.258)$.

The overall mean score of knowledge, attitude and practices was $17.37 \pm 2.23$, with scores ranging from 13 to 25 Just 2 of the respondents had good knowledge of diabetes while $9 \%$ of the respondents had poor knowledge and there was not any significant difference in the knowledge of diabetes among genders.

Attitude toward dietary modification was favorable in $74 \%$ of diabetics, but for the exercises were $48 \%$, meanwhile $47 \%$ patients believed that when diabetes is controlled, dietary management is not essential and $68 \%$ felt that insulin was the last option for treatment (Figure 1).

Table 2. Awareness of type 2 diabetes patients about diabetes care $(n=100)$.

\begin{tabular}{ll}
\hline Care in diabetes & Responses (\%) \\
\hline Diabetes is cured with & $71 \%$ \\
Healthy diet & $13 \%$ \\
Regular exercise & $5 \%$ \\
Weight control & $11 \%$ \\
Cannot prevent & \\
Symptom(s) of hypoglycemia & $10 \%$ \\
Sweating & $68 \%$ \\
Dizziness & $22 \%$ \\
Weakness/fatigue & \\
Allied Care & $25 \%$ \\
Blood sugar examination & $5 \%$ \\
Eye examination & $7 \%$ \\
Foot care for diabetics & $63 \%$ \\
Blood pressure monitoring & \\
\hline
\end{tabular}

Table 3. Level of knowledge about diabetes among type 2 diabetes patients ( $n$ $=100)$.

\begin{tabular}{llll}
\hline Level of Knowledge Score & $\begin{array}{l}\text { Poor } \\
\mathbf{n}(\%)\end{array}$ & $\begin{array}{l}\text { Average } \\
\mathbf{n}(\%)\end{array}$ & $\begin{array}{l}\text { Good } \\
\mathbf{n}(\%)\end{array}$ \\
\hline What is diabetes & $15(15 \%)$ & $71(71 \%)$ & $14(14 \%)$ \\
Etiology & $56(56 \%)$ & $30(30 \%)$ & $14(14 \%)$ \\
Sign and Symptoms & $10(10 \%)$ & $62(62 \%)$ & $28(28 \%)$ \\
Complications & $10(10 \%)$ & $72(72 \%)$ & $18(18 \%)$ \\
Medications & $82(82 \%)$ & $10(10 \%)$ & $8(8 \%)$ \\
Dietary Management & $26(26 \%)$ & $47(47 \%)$ & $27(27 \%)$ \\
Overall & $10(10 \%)$ & $79(79 \%)$ & $11(11 \%)$ \\
\hline
\end{tabular}

Table 4. Mean score of knowledge, attitude and practices of the diabetic patients based on KAP questionnaire $(n=100)$.

\begin{tabular}{lll}
\hline Variables & Mean \pm SD score & Max score \\
\hline Knowledge & $9.5 \pm 0.89$ & 13 \\
Attitude & $4.01 \pm 1.38$ & 8 \\
Practice & $2.7 \pm 1.38$ & 8 \\
Overall & $17.3 \pm 2.2$ & 29 \\
\hline
\end{tabular}

Regarding their self-reported practices during the last month only $10 \%$ of patients had a good medication adherence ( $>5$ days/week) based on doctor's prescription. Nearly $27 \%$ of the patients modified dietary habits, as prescribed by dietician. Only 33 persons (33\%) checked their foot and eye in the last 1 year by specialists.

The practices regarding physical activity and blood glucose monitoring were found to be poor (Figure 2). The most sources of information regarding diabetes were by health care personnel $(50 \%)$ followed by family members $(32 \%)$ and media (18\%).

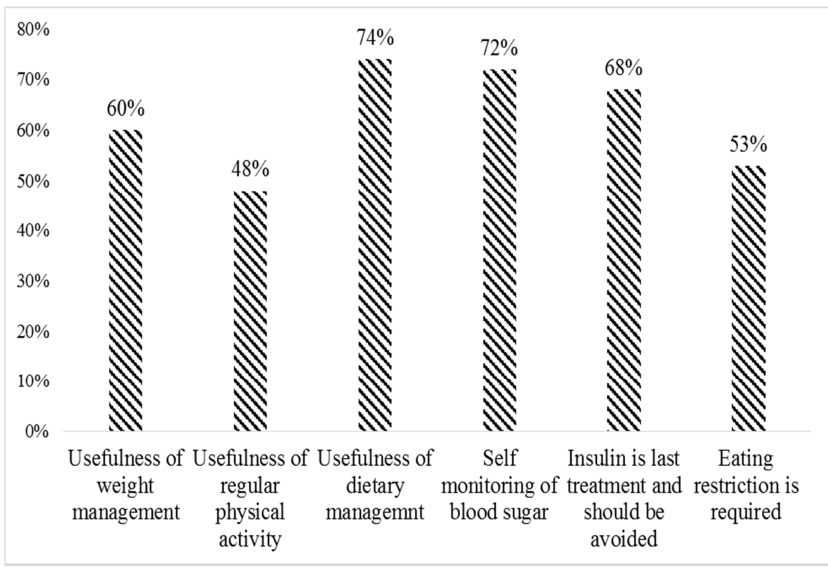

Fig. 1. Agreement in patients' attitude for diabetes control $(n=100)$.

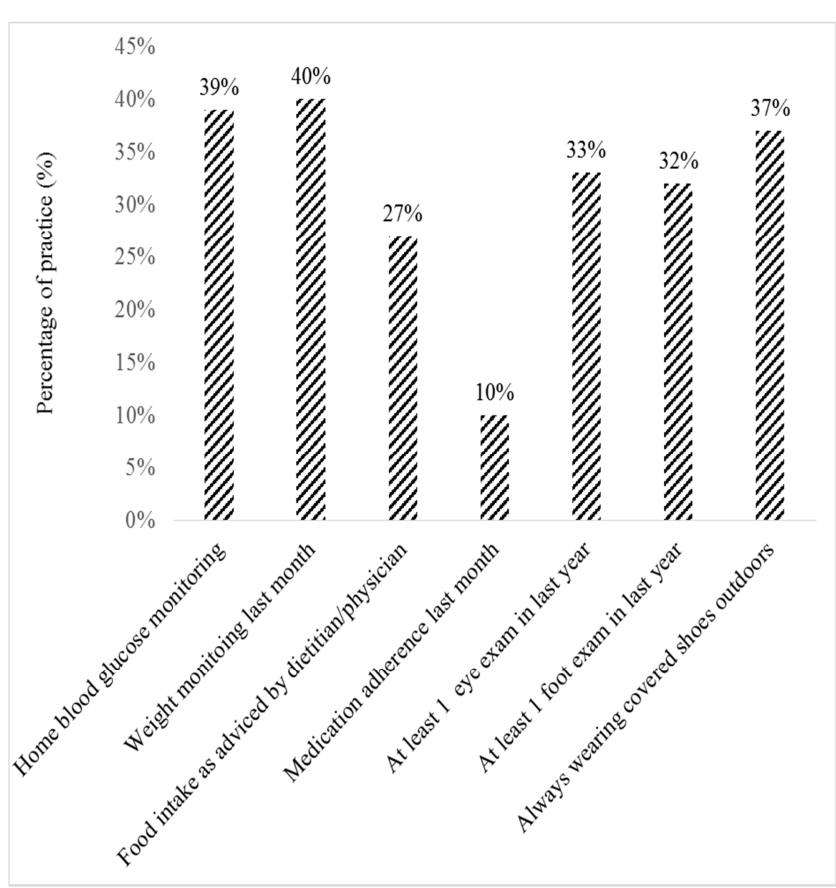

Fig. 2. Frequency of self-care practices in type 2 diabetes patients $(n=100)$.

\section{Discussion}

This cross-sectional study has been done to evaluate knowledge, attitude and practices regarding diabetes among Iranian type 2 diabetic patients in Ahvaz, Iran. Overall, it was found that diabetic patients had insufficient knowledge regarding the symptoms, complications, prevention and control of their disease condition. The KAP score for the majority of the patients was on medium level. However, a study from Malaysia reported a good knowledge, attitude and practice score among diabetic patients [17]. The differences in the results of studies may be due to the differences in educational level of the diabetic patients and accessibility of information and diabetes education.

It is well established that patient contributions are very 
important for better management of diabetes [18]. Lack of knowledge of diabetes care among patients can have adverse effects on their capabilities to control diabetes. Heart disease has been indicated as the most common complication of diabetes by $72 \%$ of the patients. Awareness of measures to identify primary complications of diabetes, such as blood pressure monitoring, periodic eye examination were poor, which reveals the need for some aspects to be focused in diabetes education programs.

Throughout knowledge assessment, we recognized that most patients didn't know about cause of diabetes and its consequences (nearly $86 \%$ ). Only a few of the patients knew that one of the common complications of diabetes is kidney disease (6\%). Insufficient awareness of complications and the importance of lifestyle modification was very obvious among patients and only $2 \%$ of them had good knowledge of diabetes.

Poor knowledge regarding diabetes has been reported in several studies from the developing countries [19, 20, 21]. While, another study in England as a developed country, also stated poor knowledge of diabetes among ethnic groups [22]. In our study, Primary education level was significantly associated with low diabetes KAP score. This may be described by the point that educated people had better access to numerous source of written informative materials.

It is a hypothesis that good KAP have impact on adequate diabetes control. However there was not any significant association between KAP score and glycemic control in this study. This result was similar the result of study among Malaysian diabetes patients [21]. Most patients in our study reported checking of blood sugar only by doctor in every three months visit; this low frequency of blood sugar self-monitoring may have effect on blood sugar control.

Awareness on lifestyle modification regarding weight management was low in this study. Some surveys have indicated that higher education was significantly associated with a better awareness [23]. The poor awareness of diabetes patients in this study might be due to the low level of education and the lack of organized diabetes education services in the diabetes clinic. Attitude regarding dietary modification and regular physical activity was acceptable in the majority. This survey discovered that diabetic patients rely mostly on drugs to control their diabetes. Therefore, better management and more effective use of health resources is very important. There are multiple barriers for the enhancement of the quality of diabetes care. These may be related to the financial statement of patients, lack of knowledge among health care providers for patient management and inadequate access to special care).

Knowledge is an essential requirement for better compliance with medical therapy [24]. Awareness of complications of diabetes was not good among the patients in this study. This may be due to the some factors such as inappropriate ways of providing information and lack of time due to the patient loads and lack of well trained staff like educators [25]. This highlights the needs for effective diabetes education programs in developing countries like Iran.
Other factors, such as health beliefs and demographic characteristics of patients have been indicated as possible links among awareness and adherence to the recommendations regarding lifestyle modifications [26].

There are not appropriate diabetes education programs in most governmental hospitals in Iran and the existing programs are weak. It has been stated that knowledge regarding diabetes among the general population and diabetics are still insufficient in Iran and a good diabetes education program is instantly required [27]. It has been also reported in a study from Pakistan that appropriate educational program can have effect on the attitude of the people about diabetes [28]. Another study showed that serious diabetes education can improve glycemic control and quality of life of diabetic patients [29, 30].

In conclusion, this study revealed a medium level of knowledge, attitude and practice for the majority of diabetes patients in Ahvaz, Iran. Diabetic patients usually are dependent on drugs for disease control while ignoring other healthy lifestyle modifications in practical. Low awareness about the diabetes among patients, affects their ability of self-management and therefore have a negative impact on outcome of diabetes. This recommends the necessity for an awareness program, patient counseling and education on self-care management of the diabetic patients to improve their knowledge regarding diabetes with the emphasis on lifestyle modifications.

\section{Acknowledgements}

No financial support for the study. No grant was given to the study. The authors declare that there is no conflict of interests.

\section{References}

[1] Shaw J, Sicree R, Zimmet P. Global estimates of the prevalence of diabetes for 2010 and 2030. Diabetes Research and Clinical Practice. 2010; 87 (1): 4-14.

[2] Adeghate E, Schattner P, Dunn E. An Update on the Etiology and Epidemiology of Diabetes Mellitus. Annals of the New York academy of sciences. 2006; 1084 (1): 1-29.

[3] World health organization: Diabetes-Factsheet; 2012. Available from: http://www.who.int/mediacentre/factsheets/fs312/en/index.ht $\mathrm{ml}$.

[4] Zimmet P, Alberti K, Shaw J. Global and societal implications of the diabetes epidemic. Nature. 2001; 414 (6865): 782-787.

[5] Esteghamati A, Gouya MM, Abbasi M, et al. Prevalence of diabetes and impaired fasting glucose in the adult population of Iran national survey of risk factors for non-communicable diseases of Iran. Diabetes Care. 2008; 31 (1): 96-98.

[6] Abdoli S, Mardanian L, Mirzaei M. How public perceive diabetes: A qualitative study. Iranian Journal of Nursing and Midwifery Research. 2012; 17(5): 370. 
[7] Diamond J. The double puzzle of diabetes. Nature. 2003; 423 (6940): 599-602.

[8] Al-Moosa S, Allin S, Jemiai N, Al-Lawati J, Mossialos E. Diabetes and urbanization in the Omani population: An Analysis of National Survey Data. Population Health Metrics. 2006; 4 (1): 5.

[9] Shetty P, Schmidhuber J. Introductory lecture the epidemiology and determinants of obesity in developed and developing countries. International Journal for Vitamin and Nutrition Research. 2006; 76 (4): 157-162.

[10] Wild S, Roglic G, Green A, Sicree R, King H. Global prevalence of diabetes estimates for the year 2000 and projections for 2030. Diabetes care. 2004; 27 (5): 1047-1053.

[11] Schramm TK, Gislason GH, Køber L et al. Diabetes patients requiring glucose-lowering therapy and non-diabetics with a prior myocardial infarction carry the same cardiovascular risk a population study of 3.3 million people. Circulation. 2008; 117(15): 1945-1954.

[12] Rhee MK, Slocum W, Ziemer DC, Culler SD, Cook CB, El-Kebbi IM, Phillips L S. Patient adherence improves glycemic control. The Diabetes Educator. 2005; 31(2): 240-250.

[13] Spann SJ, Nutting, PA, Galliher JM, et al. Management of type 2 diabetes in the primary care setting: a practice-based research network study. The Annals of Family Medicine.2006; 4(1): 23-31.

[14] Amani R, Mostafavi A (2006). Nutritional Related Lifestyle of Type 2 Diabetic Patients in Ahvaz-IR Iran. International Journal of Endocrinology and Metabolism. 2006; 3: 123-129.

[15] Abolghasemi R, Sedaghat M (2014). The Patient's attitude toward type 2 diabetes mellitus, a qualitative Study. Journal of Religion and Health. 2014;1-15.

[16] Mukhopadhyay P, Paul B, Das D, Sengupta N, Majumder R. Perceptions and practices of type 2 diabetics: A cross-sectional study in a tertiary care hospital in Kolkata. Int J Diabetes Dev Ctries. 2010; 30: 143-9.

[17] Ambigapathy R, Ambigapathy S, Ling H. A knowledge, attitude and practice (KAP) study of diabetes mellitus among patients attending Klinik Kesihatan Seri Manjung. NCD Malaysia. 2003; 2 (2): 6-16.

[18] Suppapitiporn S, Chindavijak B, Onsanit S. Effect of diabetes drug counselling by pharmacist, diabetic disease booklet and special medication containers on glycemic control of type 2 diabetes mellitus: a randomized controlled trial. Journal of the Medical Association of Thailand Chotmaihet thangphaet. 2005; 88: 134-41.
[19] Upadhyay DK, Palaian S, Shankar P. R, Mishra P, Pokhara, N Knowledge, attitude and practice about diabetes among diabetes patients in Western Nepal. Rawal Med J. 2008; 33 (1) : 8-11

[20] Gul N. Knowledge, attitudes and practices of type 2 diabetic patients. J Ayub Med Coll Abbottabad .2010; 22: 128-31.

[21] Gunay T, Ulusel B, Velipasaoglu S, Unal B, Ucku R, Ozgener N. Factors affecting adult knowledge of diabetes in Narlidere Health District, Turkey. Acta Diabetologica. 2006; 43:142147.

[22] Baradaran H, Knill-Jones R. Assessing the knowledge, attitudes and understanding of type 2 diabetes amongst ethnic groups in Glasgow, Scotland. Practical Diabetes Int. 2004; 21 (4): $143-148$.

[23] Ajlouni K, Khader YS, Batieha A, Ajlouni H, El-Khateeb M. An increase in prevalence of diabetes mellitus in Jordan over 10 years. Journal of Diabetes and its Complications. 2008; 22 (5): $317-324$.

[24] Farmer A, Kinmonth AL, Sutton S. Measuring beliefs about taking hypoglycaemic medication among people with Type 2 diabetes. Diabetic Medicine. 2006; 23(3): 265-270.

[25] Kapur A. Influence of socio-economic factors on diabetes care. Int J Diab Dev Countries. 2001; 21:77-85.

[26] Castillo EC. The effects of diabetes education intervention package with the knowledge, beliefs, attitudes, and practices, and FBS values of persons with Type 2 diabetes mellitus. University of the Philippines Manila College of Public Health. $2003 ; 125$.

[27] Delavari A, Alikhani S, Nili S, Birjandi RH, Birjandi F. Quality of care of diabetes mellitus type II patients in Iran. Archives of Iranian Medicine (AIM) .2009; 12(5): 492-495.

[28] Badrudin N, Basit A, Hydrie MZI, Hakeem R. Knowledge, Attitude and Practices of patient visiting a diabetes care unit. Pak J Nutrition. 2002; 1(2):99-102.

[29] Maina WK, Ndegwa ZM, Njenga EW, Muchemi EW. Knowledge, Attitude and Practices related to Diabetes among Community Members in Four Provinces in Kenya: A Cross-sectional study. Journal of Pan African Medical. 2011; 7 (2): 1-9.

[30] Khanna A, Bush AL, Swint JM, Peskin MF, Street Jr RL, Naik AD. Hemoglobin A1c improvements and better diabetes-specific quality of life among participants completing diabetes self-management programs: a nested cohort study. Health and Quality of Life Outcomes.2012; 10 (1): 48:1-7. 